\title{
Parameters Optimization and Energy-saving of Highway Tunnel Stagger Luminaire Distribution Lighting with LED
}

\author{
Shijuan Fan ${ }^{1, a}$, Chao Yang ${ }^{2, b}$ \\ ${ }^{1}$ School of Railway Tracks and Transportation, East China Jiaotong University, Nanchang Jiangxi, \\ China, 330013 \\ ${ }^{2}$ School of Mechatronic Engineering, East China Jiaotong University, Nanchang Jiangxi, China, \\ 330013 \\ afanshijuan_69@163.com, byangchao_69@163.com
}

Keywords: Tunnel lighting, parameters optimization, stagger-luminaire-distribution lighting, optimization model, energy-saving.

Abstract. A parameters optimization model (POM) of stagger-luminaire-distribution (SLD) lighting for tunnel interior zone was established to acquire the most energy-saving luminaire installation parameters (LIPs) of highway tunnel interior zone, with luminaire installation height, longitudinal installation spacing, crosswise installation spacing, elevation angle and luminaire power as optimization parameters, and with minimum total power consumption of the SLD lighting system as objective function. Yanlieshan tunnel of Jiujing highway was taken as example for the optimization. The optimal LIPs of the SLD lighting system of tunnel interior zone were obtained by the POM, a comparison between the optimization results and that of Yanlieshan tunnel lighting system was performed, which shows that the optimized SLD lighting system with LED lamps installed according to the optimized LIPs has remarkable energy-saving effect even under full capacity lighting condition. Illuminance and illuminance uniformity of the tunnel road surface still meet the lighting demands even the LED lamp's luminance decreases 30\%. A SLD lighting simulation experiment with the optimized SLD lighting LIPs for Yanlieshan tunnel was accomplished in the software Dialux, the simulation results basicly agree with the optimization calculation results from the POM, which proves the correctness of the SLD lighting POM.

\section{Introduction}

With rapid development of highway in China, the mileage of highway tunnels increased quickly. By the end of 2014, total length of highway tunnels in China was $10756.7 \mathrm{~km}$, and the total length of long tunnels and extra-long tunnels was $7241.6 \mathrm{~km}[1]$. Lighting facilities should be installed in the tunnels longer than 100m according to "JTG/T D70/2-01-2014. Guidelines for Design of Lighting of Highway Tunnels" of China[2]. The power consumption statistics of highway tunnels in Chongqing showed that the electricity cost per kilometer in tunnel was more than $40 \times 10^{4}$ yuan in one year[3], on the basis of this criterion, the total electricity cost of all highway tunnels in China was up to 4.3 billion yuan in 2014. The lighting system was the largest energy consumption part and its cost was huge. The huge energy consumption of tunnel lighting was the main cause of the huge operating cost of highway tunnel. Energy-saving of tunnel lighting has become a social and economic problem that can not be ignored in China. How to reduce power consumption with traffic safety has become an urgent problem needs to be solved in tunnel lighting design, construction and operation. Using energy-saving luminaires, optimizing luminaire-installation-parameters (LIPs) and intelligently controlling lighting are able to save energy of tunnel lighting. In resent years, energy-saving studies of most experts and scholars on tunnel lighting mainly focused on intelligent control of luminaires, but relatively fewer studies on optimization of LIPs.

Angel Pachamanov[4] established an optimization model of tunnel lighting and obtained the optimal LIPs with fixing luminaire power. Ren Shenhe[5] performed an energy-saving experiments of the luminaire installation height and elevation angle with LED lamps and obtained the optimal LDS. Ji Jiajun[6] performed simulations with LED lamps and HPSLs about the influences of LDS and LIPs on 
luminaire utilization factor, average illuminance and overall illuminance uniformity of tunnel road surface. But Ren Shenhe and Ji Jiajun did not establish the parameters optimization model (POM). Li Ning[7] established an intelligent optimization model of tunnel lighting based on genetic algorithms to optimize illuminance distribution of every luminaire, with minimum energy consumption of the lighting system as objective function, and he verified the optimization model with a simulation. However, fewer studies were about the installation parameters optimization of SLD lighting of highway tunnel.

A SLD lighting POM for tunnel interior zone will be established, with luminaire installation height, longitudinal installation spacing, crosswise installation spacing, elevation angle and luminaire power as optimization parameters, with minimum total power consumption of the SLD lighting system as objective function. Yanlieshan tunnel of Jiujing highway will be taken as example to be optimized with the SLD lighting POM and a SLD lighting simulation experiment with the optimized LIPs for Yanlieshan tunnel will be accomplished in software Dialux to verify the correctness of the SLD lighting POM.

\section{Lighting Formula}

Illuminance. When luminare has elevation angle $\xi$ in Y direction (Fig. 1), the horizontal illuminance at calculation point $b(x, y)$ is

$$
E_{b}=\frac{I_{c}(\gamma, \theta) h}{\left(x^{2}+y^{2}+h^{2}\right)^{1.5}}
$$

Where, $E_{b}$ is the horizontal illuminance generated by one lamp at the point $b(x, y)$ of the road surface; $h$ is the installation height of the lamp; $\gamma$ is the intersection angle of light $\mathrm{Ab}$ and optic axis $\mathrm{AO}_{1}$ of the lamp; $\theta$ is the intersection angle between the plane $\mathrm{AOC}$ and the plane $\mathrm{AbO}_{1} ; \operatorname{Ic}(\gamma, \theta)$ is the luminous intensity of the lamp in the direction of light $\mathrm{Ab}$.

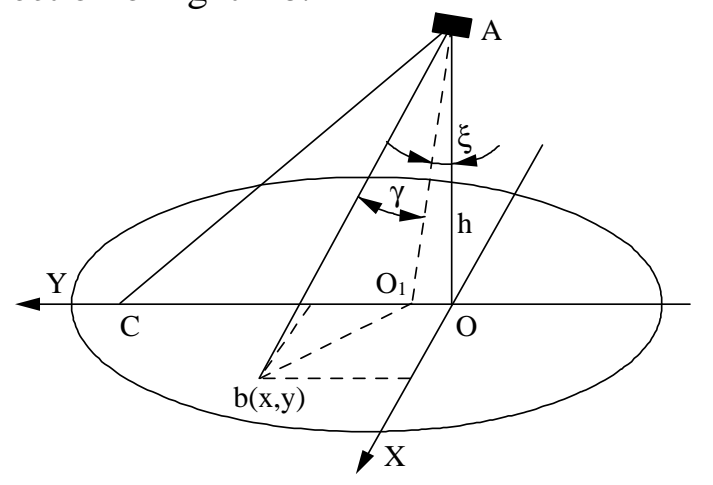

Fig. 1 Lighting schematic diagram of one lamp

Luminous Intensity of Luminaire. If photomatric data of luminaire are available, the real luminous intensity of the light-direction deviating $\gamma$ from the optical axis of the luminaire is calculated as follows

$$
\mathrm{I}_{\mathrm{c}}(\gamma, \theta)=\mathrm{I}_{1000}(\gamma, \theta) \eta \eta_{0} \mathrm{M} \Phi \times 10^{-3}
$$

Where, $I_{1000}(\gamma, \theta)$ is the luminous intensity of the luminaire from the photomatric data corresponding to $\gamma$ and $\theta ; \eta$ is light output rate of luminaire; $\eta_{0}$ is utilization factor of luminaire; $M$ is maintenance factor of luminaire; $\Phi$ is the rated luminous flux of luminaire.

\section{Parameters Optimization Model}

$\mathrm{A}_{1}, \mathrm{~A}_{2}, \mathrm{~A}_{3}$ and $\mathrm{A}_{4}$ are stagger adjacent luminaires which have the same type, same power $\mathrm{p}$, same installation height $h$ and same elevation angle $\xi$. The longitudinal installation spacing is $s$, the crosswise installation spacing is $d_{1}$, the width of the traffic lanes is $d_{0}$, the total width of the tunnel road surface 
including the width of sidewalk is $d$. The region $B_{1} B_{2} B_{3} B_{4}$ between the two adjacent luminaires $A_{2}$ and $\mathrm{A}_{3}$ is set as calculation region. A three-dimension Cartesian coordinates system can be established with the longitudinal centerline of road surface as the $\mathrm{X}$-axis, the crosswise direction as the $\mathrm{Y}$-axis, the vertical direction as the $Z$-axis, and the central point $O$ of the calculation region $B_{1} B_{2} B_{3} B_{4}$ as the original point. The schematic diagram of tunnel SLD lighting and the Cartesian coordinates system are showed in Fig. 2.

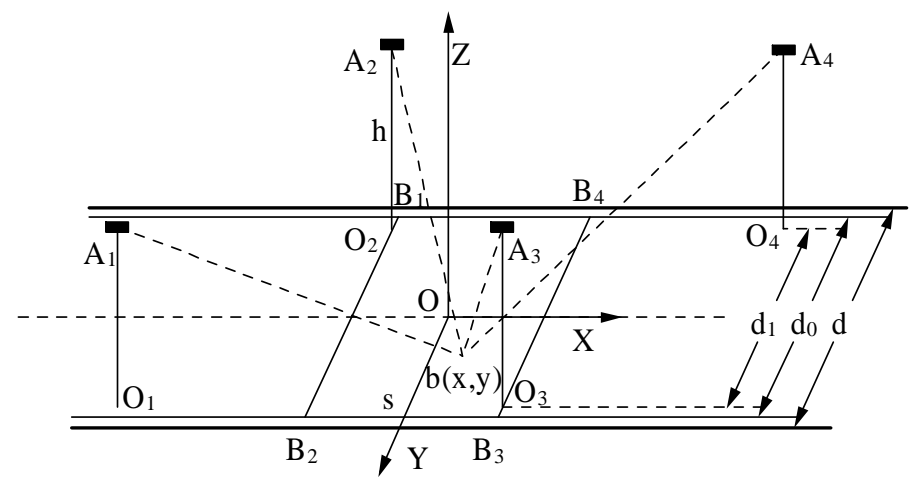

Fig. 2 Schematic diagram of tunnel SLD lighting

The overall horizontal illuminance at the calculation point $b(x, y)$ is calculated as follows

$$
\mathrm{E}_{\mathrm{b}}=\mathrm{E}_{\mathrm{b} 1}+\mathrm{E}_{\mathrm{b} 2}+\mathrm{E}_{\mathrm{b} 3}+\mathrm{E}_{\mathrm{b} 4}
$$

Where, $\mathrm{E}_{\mathrm{b} 1} \sim \mathrm{E}_{\mathrm{b} 5}$ is the horizontal illuminance at the calculation point $\mathrm{b}(\mathrm{x}, \mathrm{y})$ generated by the luminaires $\mathrm{A}_{1}, \mathrm{~A}_{2}, \mathrm{~A}_{3}$ and $\mathrm{A}_{4}$, respectively, which are calculated as follows

$$
\begin{aligned}
& E_{b 1}= \begin{cases}\frac{I_{c}\left(\gamma_{1}, \theta_{1}\right) h}{\left[(3 s / 2+x)^{2}+\left(d_{1} / 2-y\right)^{2}+h^{2}\right]^{3 / 2}}, & 3 s / 2+x \leq K\left(d_{1} / 2-y\right)+S_{1}, d_{1} / 2-y \leq S_{2} \\
0 \quad \text { others }\end{cases} \\
& E_{b 2}= \begin{cases}\frac{I_{c}\left(\gamma_{2}, \theta_{2}\right) h}{\left[(s / 2+x)^{2}+\left(d_{1} / 2+y\right)^{2}+h^{2}\right]^{3 / 2}}, & s / 2+x \leq K\left(d_{1} / 2+y\right)+S_{1}, d_{1} / 2+y \leq S_{2} \\
0 \quad \text { others }\end{cases} \\
& E_{b 3}= \begin{cases}\frac{I_{c}\left(\gamma_{3}, \theta_{3}\right) h}{\left[(s / 2-x)^{2}+\left(d_{1} / 2-y\right)^{2}+h^{2}\right]^{3 / 2}}, & s / 2-x \leq K\left(d_{1} / 2-y\right)+S_{1}, d_{1} / 2-y \leq S_{2} \\
0 \quad \text { others }\end{cases} \\
& E_{b 4}= \begin{cases}\frac{I_{c}\left(\gamma_{4}, \theta_{4}\right) h}{\left[(3 s / 2-x)^{2}+\left(d_{1} / 2+y\right)^{2}+h^{2}\right]^{3 / 2}}, & 3 s / 2-x \leq K\left(d_{1} / 2+y\right)+S_{1}, d_{1} / 2+y \leq S_{2} \\
0 \quad \text { other }\end{cases}
\end{aligned}
$$

Where, $K=\left\{\begin{array}{l}\tan (\alpha)[1-\cos (\xi)] / \sin (\xi), \quad \xi \neq 0 \\ 0, \quad \xi=0\end{array} ; S_{1}=h \tan (\alpha) ; S_{2}=h \tan (\beta+\xi) ; \alpha, \beta\right.$ is the half light-emitting angle of the luminaire in $\mathrm{X}$ direction and $\mathrm{Y}$ direction, respectively; $\gamma_{1} \sim \gamma_{4}$ is the intersection angle between the light from luminaire $A_{1} \sim A_{4}$ to calculation point $b$ and the corresponding optic axis of luminaire $A_{1} \sim A_{4}$, respectively; $\theta_{1} \sim \theta_{4}$ is the intersection angle between the cross-section $C 0 / 180$ of each luminaire and the plane composed by the light from corresponding luminaire to the calculation point $b$ and the optic axis of corresponding luminaire, respectively.

The design performance indexes of lighting system for tunnel interior zone based on the Ref. [2] are provided as follows. 
(1) Strobe frequency f should meet the demand of $\mathrm{f} \leq 2.5 \mathrm{~Hz}$ or $\mathrm{f} \geq 15 \mathrm{~Hz}$. $\mathrm{f}=\mathrm{v} / \mathrm{s}$, where, $\mathrm{v}$ is the traffic speed, $\mathrm{f}$ is set as $\mathrm{f} \leq 2.5 \mathrm{~Hz}$ for the tunnel interior zone in this work.

(2) The luminance of road surface for the tunnel interior zone should not be less than standardized minimum value under the condition of set driving speed and traffic flow.

(3) The overall and longitudinal luminance uniformity of the tunnel road surface should not be less than the values given in Ref. [2].

(4) The average illumiance of the tunnel wall area within 2 meters should not be less than $60 \%$ of the average illumiance of the tunnel road surface.

Further more, at least $0.4 \mathrm{~m}$ spacing should be reserved between the luminaire and the tunnel wall to install the lamp.

The SLD lighting POM of tunnel interior zone was established as follows

$$
\begin{aligned}
& \min \mathrm{P}=\mathrm{n} \times \mathrm{p}=\mathrm{Lp} / \mathrm{s} \\
& \text { st. }\left\{\begin{array}{l}
\mathrm{h}-\left(\mathrm{d}-\mathrm{d}_{1}\right) /[2 \tan (\beta-\xi)] \geq 2 \\
2 \mathrm{htan}(\beta+\xi) \geq \mathrm{d}_{0}+\mathrm{d}_{1} \\
\mathrm{~h}_{\text {min }} \leq \mathrm{h} \leq \mathrm{h}_{\max } \\
0 \leq \xi \leq \beta-\arctan \left[\left(\mathrm{d}-\mathrm{d}_{1}\right) /(2 \mathrm{~h}-4)\right] \\
\mathrm{v} / 2.5 \leq \mathrm{s} \leq 2 \mathrm{htan}(\alpha) / \cos (\xi)-2 \mathrm{~K}\left[\mathrm{htan}(\xi)+\left(\mathrm{d}-\mathrm{d}_{1}\right) / 2\right] \\
\mathrm{E}_{\text {min }} \geq \max \left(\mathrm{E}_{0}, \mathrm{U}_{0} \mathrm{E}_{\mathrm{av}}\right) \\
\mathrm{E}_{\text {min }} \leq \mathrm{E}_{\mathrm{av}} \\
\mathrm{E}_{\text {cmin }} \geq \mathrm{U}_{1} \mathrm{E}_{\mathrm{cmax}} \\
\mathrm{d}_{1}^{2} / 4+(\mathrm{h}-2)^{2} \leq 25
\end{array}\right.
\end{aligned}
$$

Where, $h_{\min }$ is the minimum value of the installation height of luminaire; $h_{\max }$ is the maximum value of the installation height of luminaire; $\mathrm{P}$ is the total power of $\mathrm{n}$ luminaires; $\mathrm{L}$ is the length of the tunnel interior zone; $\mathrm{p}$ is the power of one luminaire; $\mathrm{E}_{0}$ is the minimum illuminance to meet the traffic demands for tunnel interior zone; $\mathrm{E}_{\min }$ is the minimum illuminance of the tunnel road surface; $\mathrm{E}_{\mathrm{cmin}}$ is the minimum illuminance of the tunnel road surface centerline; $\mathrm{E}_{\mathrm{cmax}}$ is the maximum illuminance of the tunnel road surface centerline; $\mathrm{U}_{0}$ is the required overall luminance uniformity of the tunnel road surface; $\mathrm{U}_{1}$ is the required longitudinal luminance uniformity of the tunnel road surface centerline; $\mathrm{E}_{\mathrm{av}}$ is the horizontal average illuminance of the road surface.

\section{Optimization Example and Simulation}

Optimization Condition. Yanlieshan tunnel of Jiujing highway is taken as example, which is designed as one-way and double holes and consists of tunnel \#1 and tunnel \#2. The tunnel \#2 was selected as study object, it is $1819 \mathrm{~m}$ long and its interior zone is $1383 \mathrm{~m}$ long. The designed traffic volume is greater than 1200 vehicles per hour, designed driving speed is $80 \mathrm{~km} / \mathrm{h}$. The total width of the tunnel road is $10.25 \mathrm{~m}$, two traffic lanes in all and each is $3.75 \mathrm{~m}$ wide, clear height of the tunnel hole is $7.425 \mathrm{~m}$. There is $0.5 \mathrm{~m}$ wide marginal strip at each roadside of the tunnel, $1 \mathrm{~m}$ wide maintaining roadway at one roadside and $0.75 \mathrm{~m}$ wide maintaining roadway at the other roadside for the demand of traffic safety and maintenance[8,9]. The cross section of the tunnel hole is circular arch with radius of $5.4 \mathrm{~m}$. Based on Ref.[2], $\mathrm{U}_{0}=0.4, \mathrm{U}_{1}=0.6$, the demanded luminance value for tunnel interior zone is $3.5 \mathrm{~cd} / \mathrm{m}^{2}$. Cement concrete was adopted for the tunnel road surface, so the conversion relationship between the average illuminance and average luminance is $10 \mathrm{~lx} / \mathrm{cd} \cdot \mathrm{m}^{-2}$, so $\mathrm{E}_{0}$ is $35 \mathrm{~lx}$. In the optimization model, $\mathrm{h}_{\min }$ is $5 \mathrm{~m}$ and $\mathrm{h}_{\max }$ is $7 \mathrm{~m}$.

Light Distribution Curve and Light Intensity of Lamp. NVC LED lamps (NHLED101 103) were adopted in this optimization. Luminous efficiency of the LED lamp is $100 \mathrm{~lm} / \mathrm{W}$, the light-emitting angle is $120^{\circ} \times 120^{\circ}, \eta=1$, the light distribution curve (LDC) of the LED lamp is showed in Fig. 3. It is can be observed that the LDC in cross-section C0/180 is close to the LDC in cross-section C90/270, so 
the LDC of the LED lamp was treated as full axial symmetric for simplified calculation. M was set 0.7, $\eta_{0}$ can be up to $0.8 \sim 0.913[10,11], 0.85$ was assigned to $\eta_{0}$ in this optimization.

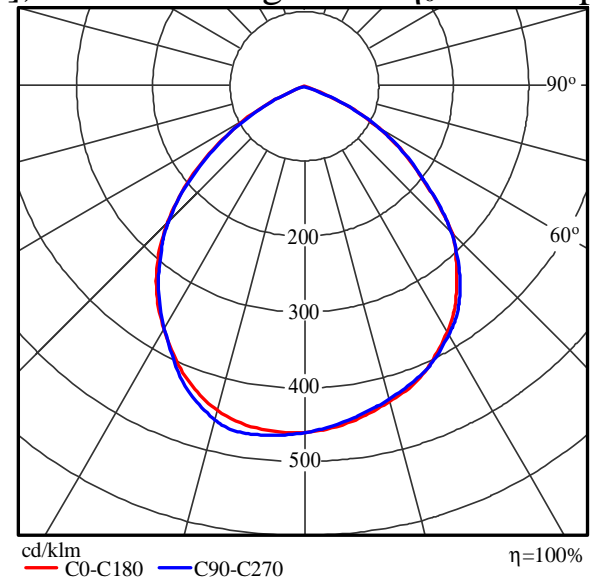

Fig. 3 Light distribution curve of LED lamp

The real luminous intensity of the point $b(x, y)$ in the calculating region of the tunnel road surface generated by one LED lamp is calculated as follows

$$
I_{c}(\gamma, \theta)=46 \eta_{0} \eta \operatorname{Mpf}(\gamma)
$$

Where, $f(\gamma)$ is the fitted luminous intensity coefficient of light deviating (changing from 0 to $\pi / 2$ ) from the light axis of LED lamp, which is obtained based on the $\mathrm{I}_{1000}(\gamma, \theta)$ of the LED lamp.

$$
\mathrm{f}(\gamma)=0.999 \mathrm{e}^{-\left(\frac{\gamma-0.06845}{0.6399}\right)^{2}}+0.3048 \mathrm{e}^{-\left(\frac{\gamma-0.7187}{0.3406}\right)^{2}}
$$

Optimization of LIPs and Analysis. Optimized LIPs of SLD lighting with LED lamps in the tunnel interior zone were obtained by substituting the known parameters in the POM and listed in Table 1. It can be seen from Table 1 that the lighting system of tunnel interior zone can save most energy while $\xi=0^{\circ}$ and $d_{1}=0$, that is, the central LDS is the most energy-saving. In order to study the influence of crosswise installation spacing, $\mathrm{d}_{1}$, on other LIPs and total power consumption, optimized parameters of SLD lighting with different fixed crosswise installation spacing were obtained and listed in Table 2. It can be seen from Table 2 that the power of one lamp and total power consumption increase with increasing of $\mathrm{d}_{1}$ in the range from $1 \mathrm{~m}$ to $5 \mathrm{~m}$. No optimal solution could be obtained from the POM when $d_{1}$ was greater than $5 \mathrm{~m}$.

Table 1 Optimized parameters of SLD lighting

\begin{tabular}{cccccc}
\hline Parameters & $\xi\left[{ }^{\circ}\right]$ & $\mathbf{s}[\mathbf{m}]$ & $\mathbf{H}[\mathbf{m}]$ & $\mathbf{p}[\mathbf{W}]$ & $\mathbf{P}[\mathbf{k W}]$ \\
\hline Optimized results & 0 & 8.89 & 7 & 107 & 16.692 \\
\hline
\end{tabular}

Table 2. Optimized parameters of SLD lighting with different fixed crosswise spacing

\begin{tabular}{ccccccc}
\hline $\mathrm{d}_{1}[\mathrm{~m}]$ & $\mathrm{s}[\mathrm{m}]$ & $\mathrm{h}[\mathrm{m}]$ & $\xi\left[^{\circ}\right]$ & $\mathrm{p}[\mathrm{W}]$ & $\mathrm{n}$ & $\mathrm{P}[\mathrm{kW}]$ \\
\hline 1 & 8.89 & 6.97 & 3 & 109 & 156 & 17.004 \\
2 & 8.90 & 6.90 & 8.5 & 115 & 156 & 17.94 \\
3 & 9.41 & 6.77 & 13 & 130 & 147 & 19.11 \\
4 & 9.77 & 6.58 & 17.5 & 145 & 142 & 20.59 \\
5 & 8.89 & 6.53 & 32 & 135 & 156 & 21.06 \\
\hline$>5$ & No optimal solution & & & & \\
\hline
\end{tabular}

Comparisons between the optimized LDS (with LED lamps, $\mathrm{d}_{1}=5 \mathrm{~m}, \mathrm{~s}=9.77 \mathrm{~m}, \mathrm{~h}=6.58 \mathrm{~m}, \mathrm{p}=145 \mathrm{~W}$ and $\xi=17.5^{\circ}$ ) with the original LDS (with HPSLs) and the transformed LDS with 
electromagnetic induction lamps (EILs) were listed in Table 3. It can be seen from Table 3 that the original HPSL LDS consumed most electric energy, EIL LDS can save 20\% electric energy than the HPSL LDS only by replacing the 100W HPSLs with 80W EILs, and the optimized SLD lighting LDS with 145W LED lamps can save more 25\% electric energy than the EIL LDS and can save more $40 \%$ electric energy than the LDS with HPSLs even the optimized LED LDS works at full capacity. The energy-saving effect is remarkable.

Table 3 Total power consumption comparisons of three LDSs

\begin{tabular}{cccccc}
\hline Luminaire & LDS & s [m] & n & p[W] & P [kW] \\
\hline LED & stagger & 9.77 & 142 & 145 & 20.59 \\
EIL[16] & symmetrical & 8 & 346 & 80 & 27.68 \\
HPSL & symmetrical & 8 & 346 & 100 & 34.60 \\
\hline
\end{tabular}

Simulation Verification of Optimized Results. A simulation tunnel model was built in software Dialux based on the structural parameters of Yanlieshan tunnel of Jiujing highway and a SLD lighting system was established in the tunnel model based on the optimal LIPs. NVC NHLED103 150W/120 was adopted in the simulation model. For the LED lamp, p is $150 \mathrm{~W}, \eta$ is $100 \mathrm{~lm} / \mathrm{W}$, the light-emitting angle is $120^{\circ} \times 120^{\circ}$. In the simulation model, $\mathrm{s}$ is $9.77 \mathrm{~m}, \mathrm{~h}$ is $6.58 \mathrm{~m}, \mathrm{~d}_{1}$ is $4 \mathrm{~m}$ and $\xi$ is $17.5^{\circ}$. The calculation region was divided into $10 \times 10$ small regions in $\mathrm{X}$ direction and $\mathrm{Y}$ direction, the simulated illuminance values of the tunnel road surface $(11 \times 11$ calculation points in all $)$ obtained by the simulation lighting system were shown in Fig. 4.

$\begin{array}{rrrrrrrrrrrr}\mathbf{8 . 5 0 0} & 55 & 54 & 54 & 53 & 55 & 60 & 68 & 79 & 90 & 97 & 98 \\ \mathbf{7 . 6 5 0} & 79 & 78 & 77 & 76 & 78 & 83 & 93 & 106 & 120 & 130 & 133 \\ \mathbf{6 . 8 0 0} & 93 & 93 & 90 & 87 & 87 & 91 & 102 & 117 & 132 & 144 & 149 \\ \mathbf{5 . 9 5 0} & 111 & 111 & 106 & 100 & 96 & 99 & 108 & 123 & 138 & 151 & 158 \\ \mathbf{5 . 1 0 0} & 130 & 130 & 123 & 112 & 104 & 103 & 110 & 122 & 137 & 150 & 157 \\ \mathbf{4 . 2 5 0} & 148 & 148 & 137 & 120 & 108 & 105 & 109 & 117 & 129 & 141 & 148 \\ \mathbf{3 . 4 0 0} & 160 & 160 & 145 & 124 & 109 & 102 & 103 & 108 & 116 & 125 & 132 \\ \mathbf{2 . 5 5 0} & 161 & 159 & 142 & 121 & 105 & 96 & 94 & 96 & 101 & 108 & 113 \\ \mathbf{1 . 7 0 0} & 149 & 146 & 130 & 112 & 97 & 88 & 83 & 84 & 87 & 91 & 94 \\ \mathbf{0 . 8 5 0} & 130 & 127 & 115 & 101 & 88 & 78 & 73 & 73 & 74 & 77 & 79 \\ \mathbf{0 . 0 0 0} & 96 & 93 & 85 & 73 & 63 & 55 & 52 & \underline{51} & 52 & 53 & 54 \\ \mathbf{m} & \mathbf{0 . 0 0 0} & \mathbf{0 . 9 7 7} & \mathbf{1 . 9 5 4} & \mathbf{2 . 9 3 1} & \mathbf{3 . 9 0 8} & \mathbf{4 . 8 8 5} & \mathbf{5 . 8 6 2} & \mathbf{6 . 8 3 9} & \mathbf{7 . 8 1 6} & \mathbf{8 . 7 9 3} & \mathbf{9 . 7 7 0}\end{array}$

Fig.4 Simulated illuminance values of the calculation region points from Dialux

The simulated illuminance data of the tunnel road surface with 145W LED lamps could be obtained by multiplying the data in Fig. 4 by (145/150), the illuminance and illuminance uniformity were listed in Table 4, the data in round brackets were illuminance values after LED lamp's luminance decreases $30 \%$.

Table 4 Illuminance and illuminance uniformity of the tunnel road surface with optimized SLD lighting parameters

\begin{tabular}{ccccccc}
\hline & $\mathrm{E}_{\min }[\mathrm{lx}]$ & $\mathrm{E}_{\mathrm{cmin}}[\mathrm{lx}]$ & $\mathrm{E}_{\mathrm{cmax}}[\mathrm{lx}]$ & $\mathrm{E}_{\mathrm{av}}[\mathrm{lx}]$ & $\mathrm{U}_{0}$ & $\mathrm{U}_{1}$ \\
\hline \multirow{2}{*}{ Optimized results } & $50.01(35.01)$ & $96.47(67.53$ & $128.9(90.2$ & $93.11(65.17$ & 0.53 & 0.74 \\
& & ) & $2)$ & ) & 7 & 8 \\
Simulated results & $49.3(34.51)$ & $101.5(71.05$ & $143.1(100$. & $101.27(70.9$ & 0.48 & 0.70 \\
Standard demands[2] & $\geq 35$ & & $1)$ & ) & 7 & 9 \\
\end{tabular}

As seen from Table 4, if the optimal SLD lighting system was adopted, the optimization calculation illuminance and illuminance uniformity of the tunnel road surface and the simulated illuminance and illuminance uniformity of the tunnel road surface both met tunnel lighting demands before the LED lamp's luminance decreased 30\%. When the LED lamp's luminance decreased 30\%, only the simulated minimum illuminance was less than standard demand, but all other illuminance values met tunnel 
lighting demands. The error between the simulated minimum illuminance and the optimization calculation minimum illuminance of the tunnel road surface is less than $1.5 \%$, other simulated illuminance values are greater than optimization calculation illuminance values. The causes of error are: (1) LDC of the LED lamp was approximately symmetrical, but they were treated as full axial symmetrical in the POM; fitted luminous intensity coefficient was used to calculate the illuminance of tunnel road surface in the POM, but photomatric data of the LED lamp were directly used to calculate the illuminance of tunnel road surface in simulation. (2) a part of light was reflected to the tunnel road surface by the tunnel walls in simulation, but light reflection was not considered in the POM, which led to simulation results greater than optimization calculation results. Optimization calculation results basicly agreeing with the simulated results verifies the correctness of the POM.

\section{Summary}

A SLD lighting POM for tunnel interior zone was established. Yanlieshan tunnel of Jiujing highway was taken as example and the optimal SLD lighting LIPs of the tunnel interior zone were obtained by the POM. The optimization calculation illuminance and illuminance uniformity of the tunnel road surface both meet the tunnel lighting demands when the LED lamp's luminance decrease is less than $30 \%$ of its original luminance. Compared with the original LDS with HPSLs and the transformed LDS with EILs, the energy-saving effect of the optimized SLD lighting with LED lamps is remarkable even the optimized SLD lighting system works at full capacity.

A simulation experiment for the optimized SLD lighting was completed in the software Dialux, the simulation results basicly agree with the optimization calculation results from POM, which proves the correctness of the SLD lighting POM.

\section{Acknowledgements}

This work was financially supported by the National Natural Science Foundation of China (61463015).

\section{References}

[1] Information on http://www.zgjtb.com/2015-04/30/content_27060.htm.

[2] Ministry of Transport of the People's Republic of China: JTG/T D70/2-01-2014. Guidelines for Design of Lighting of Highway Tunnels. (China Communications Press, China, 2014). (in Chinese)

[3] Shi Lingna,Tu Yun,Wang Xiaojun: China Illuminating Engineering Journal, (2015),No.1, p.50-54. (in Chinese)

[4] Angel Pachamanov, Dessislava Pachamanova: Engineering Optimization, (2008),No.1, p.47-65.

[5] Ren Shen-he, Han Kai-xuan: Internet of Things Technologies, (2012),No.7, p30-32. (in Chinese)

[6] Ji Jiajun:The research on parameter optimization of energy-saving illumination in three-lane highway tunnel.(Chongqing Jiaotong University, China, 2012) (in Chinese)

[7] LI Ning, WANG Xiaodong, WU Jiande,etc.: Journal of Central South University (Science and Technology), Vol.14(2013) ,S, p.342-345.(in Chinese)

[8] He Lingjian, Li Shijing: Exploration Engineering(Rock \& Soil Drilling and Tunneling), (1999),No.6, p.41-43. (in Chinese)

[9] WU Jiezhen: Exploration Engineering (Rock \& Soil Drilling and Tunneling), (2003), S1, p.295-298. (in Chinese)

[10] Sagawa K, Takeichi K: Light \& Vis. Env. Vol.11(1987),No.1, p.22-29. 
[11] Yang Chao, Wang Zhiwei: China Illuminating Engineering Journal, Vol.22(2011), No.2, p.60-66. (in Chinese)

[12]Chen Wencheng, Chen Dahua, Chen Yuming. etc.: China Illuminating Engineering Journal, Vol.16(2005), No.1, p.51-55. (in Chinese) 